BY acceptance of this article, the publisher or recipient acknowledges the U.S. Government's right to retain a noriexclusive, rovalty free license in and to any copyright covering the articlo.

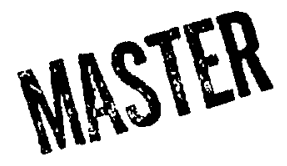

PHYSICS OF FUSION-FUEL CYCLES *

J. Rand McNally, Jr.

Fusion Energy Division

Oak Ridge National Laboratory

Oak Ridge, Tennessee 37830

Invited Paper Vugraphs

1981 IEEE International Conference on

Plasma Sclence

May 18-20, 1981

Santa $\mathrm{Fe}$, NM

*Research sponsored by the Office of Fusion Energy, U.S. Departmcat of Energy, under contract W-7405-eng-26 with the Union C rbide Corporation. 


\section{NOTES ON FIGURES}

1. Title page.

2. Abstract of paper.

3. Nuclear fusion fuels.

4. Fusion fuel costs.

5. Normalized churged particle output power for various "pure" fusion fuels opercting at $\mathrm{n}_{e}=10^{20} \mathrm{e} / \mathrm{m}^{3}$ and $n_{i} / n_{j}=z_{j} / z_{1}$. For neutron producing fuels the total power output is larger (e.g., factor of us for catalyzed $D-T, \sim 1.6$ for catalyzed $D-D)$.

6. Bibliography of useful reaction tables and graphs of 〈ov> vs $T$.

7. Progress in thermal energy uttlization factor, $f$, for toroldal devices vs year. Fast fusion factor, $\varepsilon$, for 1978 PLT point would improve $Q(=D T$ fusion power/plasma power throughput) by about a factor of 3. Consult Nuc1. Fusion 17, 1273 (1977); ORNL/TM-6362, July 1978 .

8. Progress in $f$ for mirrors and toroidal devices vs year. Exponential build-up of proton density in mirrors has not been exploited fully.

9. Approximate evaluation of tritium burnup vs $n \tau$ and $T$ demonstrating need for large $n \tau$ and high $\mathrm{T}$ to reduce tritium recycle.

10. Energy transfer from an ion to a Maxwellian sea of electrons at $T_{1}=10 \mathrm{keV}$ for various electron velocities. Note that the dominant energy loss is to electrons which are slower than the test ion.

11. Rosenbluth depletion effect for pumping electrons out of. "slow" region and thus reducing stopping power.

12. Effect of magnetic fleld cn stopping power of electrons for a test ion roving parallel to magnetic field.

13. Slowing down rates for fast fusion product ions due to nuclear elastic collisions (after Devaney and Stein).

14. Comparison of nuclear elastic slowing down rate and Coulomb (FP evergy to ions and electrons) slowing down rates in deuteron plasma at $n_{e}=n_{d}=10^{14}$ particles $/ \mathrm{cm}^{3}$. I designates test ion, Ar designates reduced atomic mass number. 
15. Bibliography of useful nuclear data information.

16. Probability of propagation chain reaction in pure DT burn vs plasma electron temperature. Actual proton consumptirn depends on fuel/ash mix but may be about $10 \%$.

17. Effects of magnetic field correction, Rosenbluth effect, nuclear scattering correction and $\mathrm{p}+\mathrm{t} \rightarrow \mathrm{n}+{ }^{3} \mathrm{He}$ reactions on plasma reactivity.

18. Tritium production in and required tritium breeding ratios for DT reactors having depleted tritium. Excess neutrons can be used for energy multiplication or fissile fuel breeding. Catalyzed DD burn would have about $1-2 \%$ tritium.

19. Advantages and disadvantages of catalyzed DD reactors. Such reactors are probably the most promising for a viable fusion economy Grossly reduced tritium inventory, no Li breeding region.

20. Nuclear effects in fusion plasmas. Further study of these phenomena may add or detract from present projections of fusion reactivity.

21. Fast proton reactions with ${ }^{6} I_{i} i$ in high temperature reactors, $\sigma v_{p}\left(p,{ }^{3} \mathrm{He}\right)$ is ov for $\mathrm{p}+{ }_{*}^{6} \mathrm{Li} \rightarrow{ }^{3} \mathrm{He}+\alpha+4.0 \mathrm{MeV}$; $\sigma v\left(\mathrm{p}, \mathrm{p}^{\prime}\right)$ is $\sigma v^{\mathrm{P}}$ for $\mathrm{p}+{ }^{6} \mathrm{Li} \rightarrow \mathrm{p}^{-}+{ }^{6} \mathrm{Li}^{*}-2.2 \mathrm{MeV} \rightarrow \mathrm{p}^{-}+\mathrm{d}+\alpha-1.7 \mathrm{MeV}$. Fast fusion reaction probability curves for $\mathrm{p}\left({ }^{6} \mathrm{Li},{ }^{3} \mathrm{He}\right) \alpha$ and $p\left({ }^{6} \mathrm{Li}, \mathrm{p}^{\prime}\right)^{6} \mathrm{Li}$ are shown for several electron temperatures. Electron temperatures will probably not exceed $150 \mathrm{keV}$ in realistic plasmas.

22. Possibility of in situ ICRH coupling between fusion product charged particles and fuel ions.

23. Status of $p-1 l_{B}$ fusion fuel prospects.

24. Radioactive ${ }^{7}$ Be production in enriched ${ }^{1 l_{B}}$ fuel $\left(97 \% 11_{B}, 10 \% 10_{B}\right)$.

25. Problem of thermal and density excursions in ignited plasmas.

26. Summary. 
PHYSICS OF FUSION FUEL CYCLES*

J. Rand McNally, Jr.

Fusion Energy Division

Oak Ridge National Laboratory

Oak Ridge, TN 37830

\section{IEEE Ir ternational Conference on \\ Plasma Science \\ May $18-20,1981$ \\ Santa $\mathrm{Fe}$, NM}

*Research sponsored by the Office of Fusion Energy, U.S. Department of Energy, under contract W-7405-eng-26 with the Union Carbide Corporation. 


\section{Physics of Fusion Fuel Cycles. J. RAND}

McNALLY, Jr., Oak Ridge National Laboratory. * The evaluation of nuclear fusion fuels for a magnetic fusion economy must take into account the various technological impacts of the various fusion fuel cycles as well as the relative reactivity and the required $B^{\prime} s$ and temperatures necessary for economic steady-state burns. This paper will review some of the physics of the various fusion fuel cycles ( $D-T$, catalyzed D-D, D- ${ }^{3} \mathrm{He}, \mathrm{D}-{ }^{6} \mathrm{Li}$, and the exotic fuels: ${ }^{3} \mathrm{He}{ }^{3} \mathrm{He}$ and the proton-based fuels such as $\mathrm{P}^{-6} \mathrm{Li}$, $\mathrm{P}-{ }^{9} \mathrm{Be}$, and $\mathrm{P}-{ }^{1}{ }^{\mathrm{B}} \mathrm{B}$ ) including such items as:

1) Tritium inventory, burnup, and recycle, 2) Neutrons, 3) Condensable fuels and ashes, 4) Direct eiectrical recovery prospects, 5) Fissile breeding, etc. The advantages as well as the disadvantages of the different fusion fuel cycles will be discussed. The optimum fuel cycle from an overall standpoint of viability and potential technological considerations appears to be catalyzed D-D, which could also support smaller relatively "clean", lean-D, rich- ${ }^{3}$ He satellite reactors 1,2 as well as fission reactors. ${ }^{3}$

* Research sponsored by the Office of Fusion Energy U.S. Department of Energy, under contract W-7405-eng-26 with the Union Carbide Corporation.

${ }^{1}$ G. H. Miley et al., EPRI ER-536-SR (1977), p. 39.

$2 \mathrm{~J}$. Rand McNally, Jr., Nucl. Fusion 18, 133 (1978).

${ }^{3}$ M. J. Saltmarsh, W. R. Grimes, R. T. Santoro, ORNL/PPA-79/3 (1979). 
NUCLEAR FUSION FUELS

"Classical" Fusion Fuels

$$
\begin{aligned}
& \text { DT - } 20 \% \text { charged particles, } 80 \% 14 \mathrm{MeV} \text { n's. } \\
& \text { Must breed } T \text { from Li (DT-Li reactor). } \\
& \text { Radioactive T ( } 2100 \text { megacuries). }
\end{aligned}
$$

"Conventional" Advanced Fusion Fuels

DD Practical advanced fusion fuels for steady-state, $\left.D^{6} L_{i}\right\}$ moderate 8 plasmas.

$D^{3} \mathrm{He}$ Relatively "clean" fuel burn.

Dependent on $n-T$, or DD or $D^{6} L i$ economy.

"Exotic" Advanced Fusion Fuels

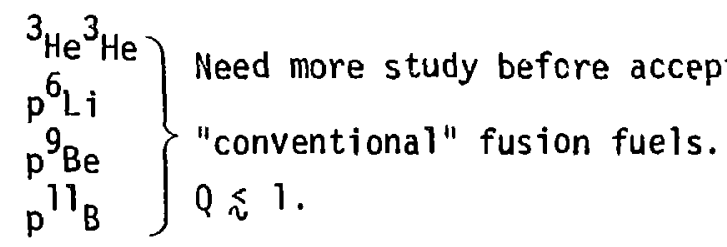


FUSION FUEL $\operatorname{costs}^{1}, 2$

\begin{tabular}{|c|c|c|c|c|}
\hline Fuel & Supplier & Purity & Cost & $\begin{array}{l}\text { Unit Fuel } \\
\text { Cost (FBU }=1.0)\end{array}$ \\
\hline D & S.R.I. & $99.1 \%$ & $1063 \$ / \mathrm{kg}$ & $0.008 \mathrm{mil} / \mathrm{kwth} \mathrm{hr}$. \\
\hline $\mathrm{T}$ & M.I. & $(>94 \%)$ & $7.5 \times 10^{6}$ & 42. \\
\hline${ }^{3} \mathrm{He}$ & M.L. & 99.9 & $7.35 \times 10^{5}$ & 4.5 \\
\hline${ }^{6} \mathrm{~L} 1$ & O.R.N.L. & 95 & 1250 & 0.03 \\
\hline $11_{B}$ & E.P. & 97 & 36,000 & 1.7 \\
\hline
\end{tabular}

${ }^{1}$ DOE established prices provided by J。 Ratledge, C. Benson (ORNL).

${ }^{2}$ Prices and purities subject to revision based on demand and technological improvements. 


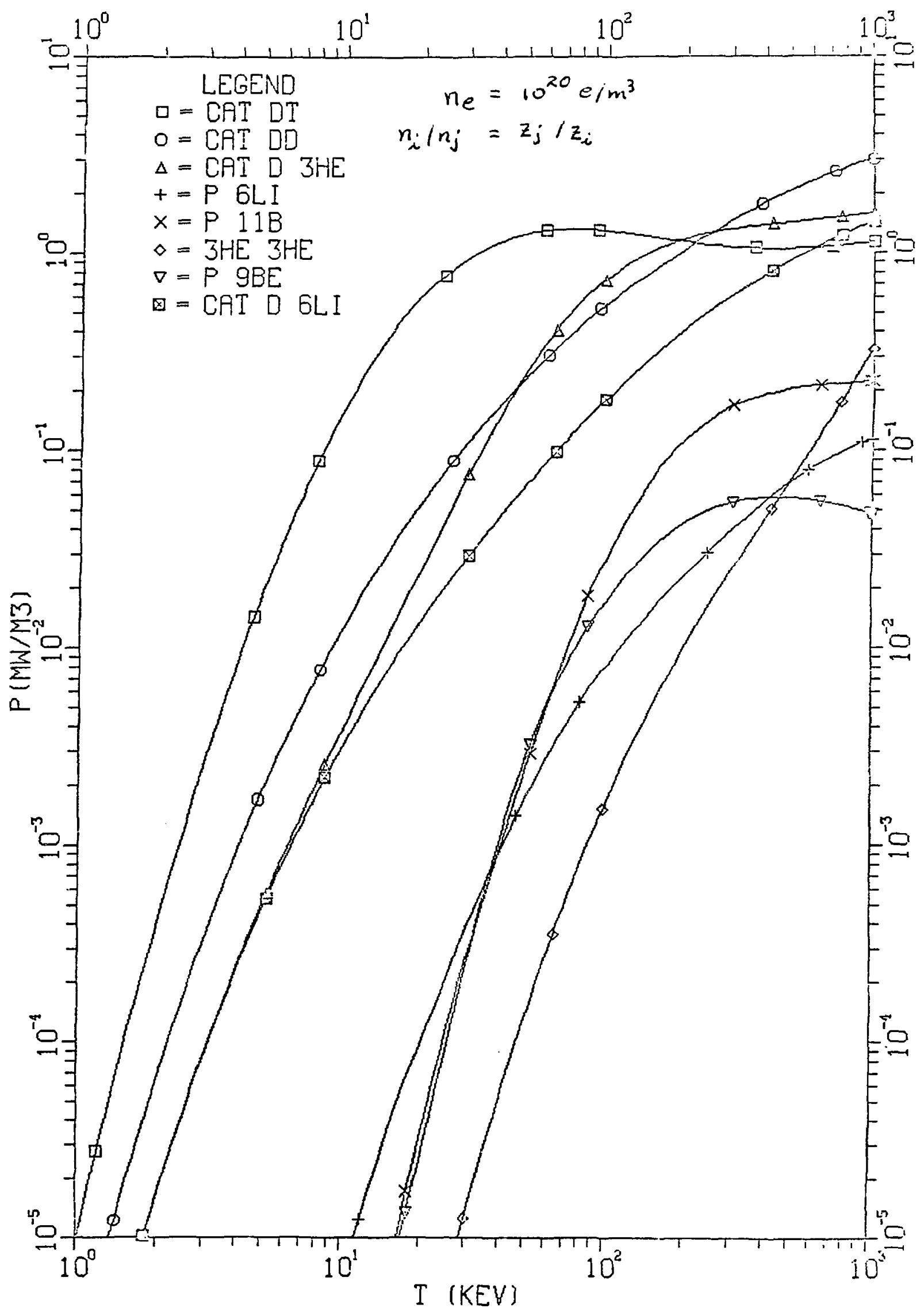

Fig. 5 


\section{REACTION TABLES AND GRAPHS}

1. J. R. McNally, Jr., K. E. Rothe, R. D. Sharp,

"Fusion Reactivity Graphs and Tables for

Charged Particle Reactions," ORNL/TM-6914

(1979); update October, 1980. (37 reactions).

2. R. J. Howerton, "Maxwel1-Averaged Reaction

Rates $(\sigma \bar{v})$ for Selected Reactions between

Ions with Atomic Mass $\leq 11, "$ UCRL-50400, 21,

Part A (1979). (24 reactions). 
SUBSTANTIAL PROGRESS

HAS BEEN MADE IN ACHIEVING

THE CONDITIONS NECESSARY FOR FUSION

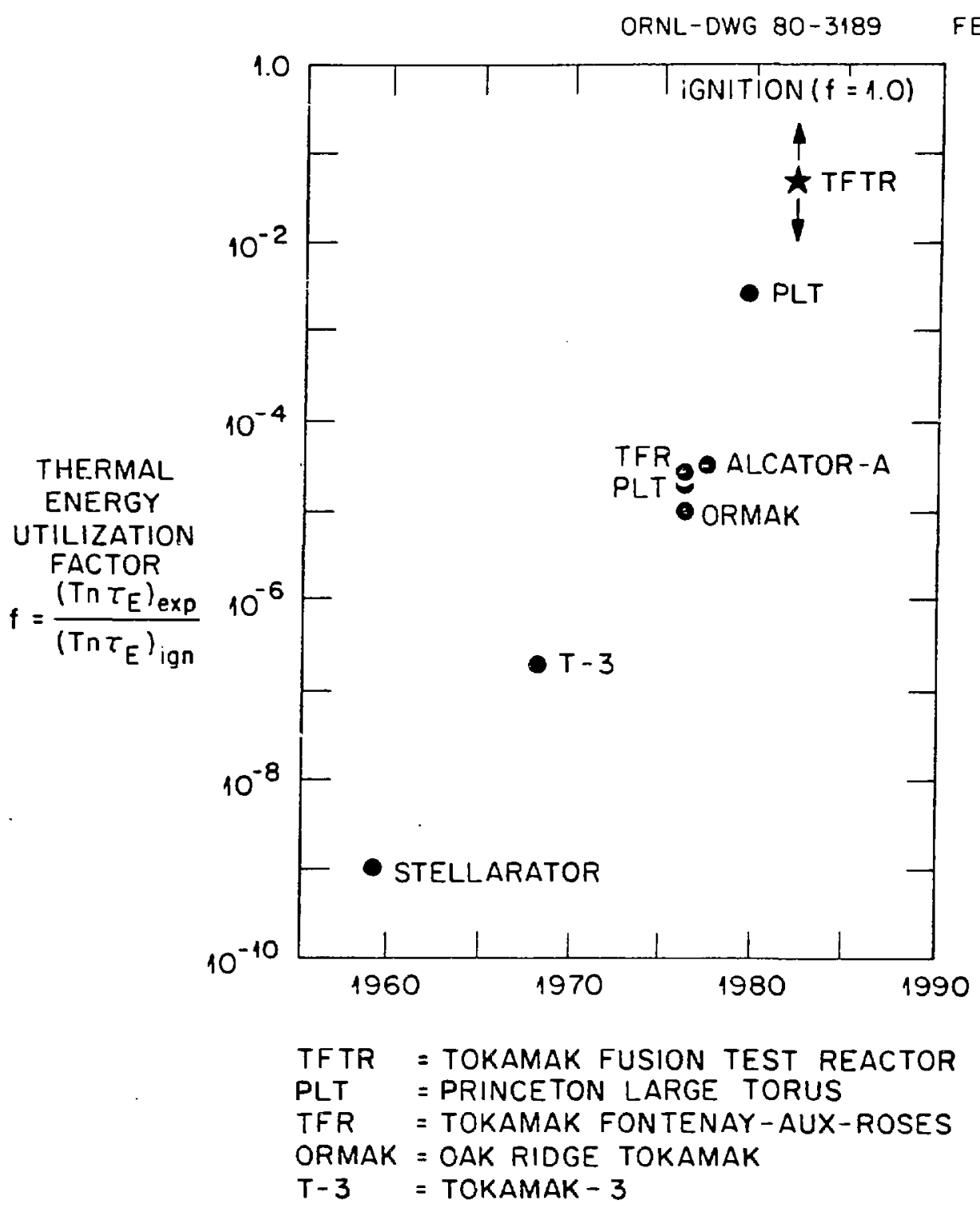


ENERGY UTILIZATION FACTOR, \& VERSUS YEAR

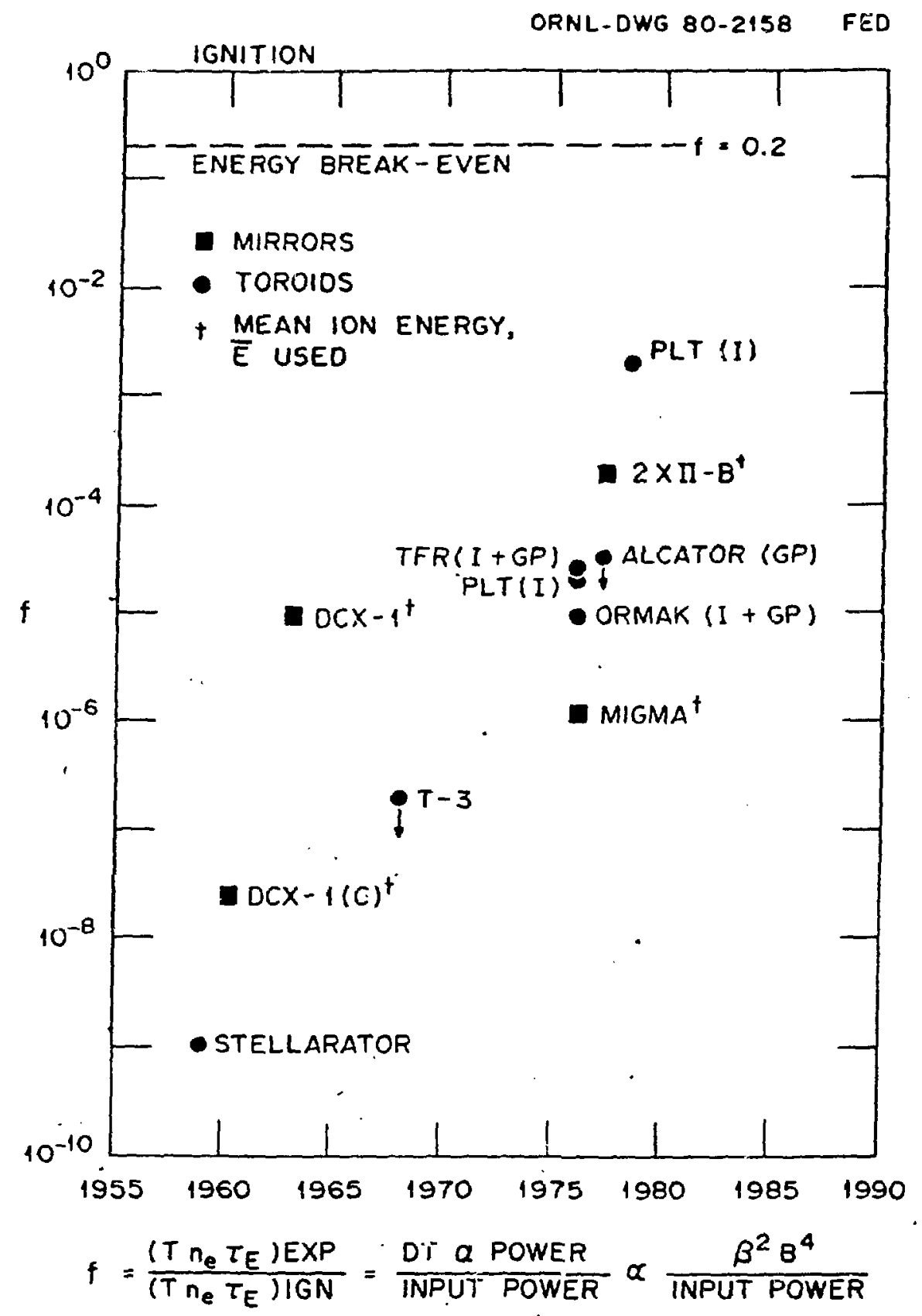

FIg. 8 
TRITIUM BURN-UP

$$
\begin{gathered}
S_{T}=\frac{n_{T}}{\tau_{T}}+n_{D} n_{T}<\sigma V> \\
F B T=\frac{n_{D} n_{T}<\sigma v>}{s_{T}}=\frac{n_{D} \tau_{T}<\sigma V>}{1+n_{D} \tau_{T}<\sigma V>}
\end{gathered}
$$

\begin{tabular}{|c|c|c|c|c|}
\hline $\begin{array}{c}{ }^{n_{D}{ }^{\tau} T} \\
\left(\mathrm{~cm}^{-3} \mathrm{sec}\right)\end{array}$ & $\begin{array}{l}T= \\
\langle\sigma v\rangle=\end{array}$ & $\begin{array}{c}10 \mathrm{keV} \\
1.1 \times 10^{-16}\end{array}$ & $\begin{array}{c}20 \mathrm{keV} \\
4.3 \times 10^{-16}\end{array}$ & $\begin{array}{l}30 \mathrm{keV} \\
6.7 \times 10^{-16} \mathrm{~cm}^{3} \mathrm{sec}^{-1}\end{array}$ \\
\hline \multicolumn{2}{|c|}{$3 \times 10^{14}$} & 0.03 & 0.11 & 0.17 \\
\hline \multicolumn{2}{|c|}{$6 \times 10^{14}$} & 0.06 & 0.20 & 0.29 \\
\hline \multicolumn{2}{|c|}{$1 \times 10^{15}$} & 0.10 & 0.30 & 0.40 \\
\hline
\end{tabular}

Note: For 50:50 DT mixture $n \tau \simeq 2 n_{D} \tau T$ 
ORNL-DWG $76-15,413$

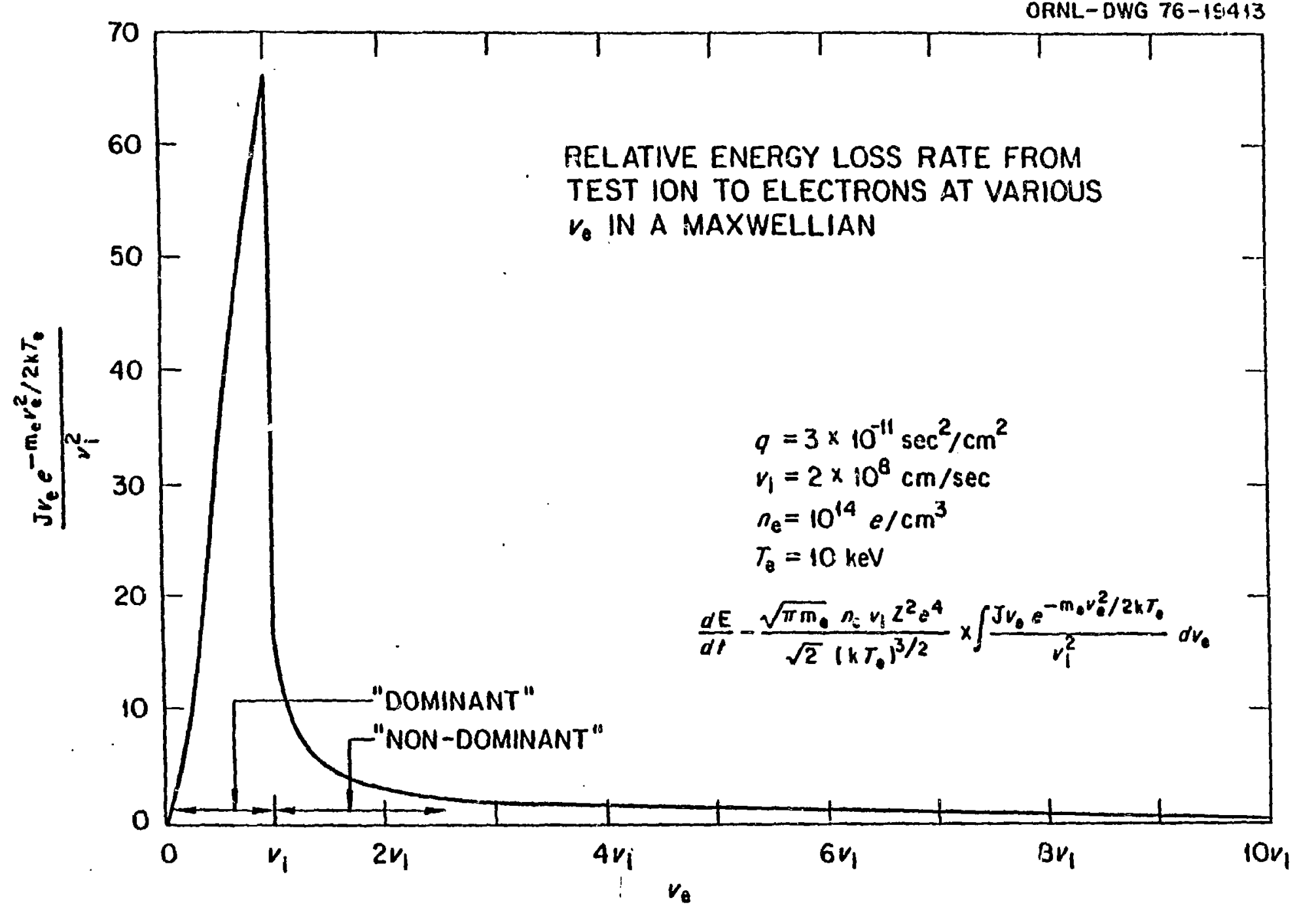


EFFECT OF ROSENBLUTH CORRECTION FOR DEPLETION OF COLD ELECTRONS IN STEADY-STATE CATALYZED DD PLASMAS

$$
\text { Input }\left\{\begin{array}{l}
B=5.0 \mathrm{~T}, R_{\mu}=0.9, \quad a=5 \mathrm{~m}, 0-D \\
n_{e}=1.0 \times 10^{14} \mathrm{~cm}^{-3}, n_{d} / n_{e}=0.55
\end{array}\right.
$$

$$
\left.\left.\frac{d E}{d t}\right)_{i-e}=\frac{d E}{d t}\right)_{\text {spitzer }} \times\left[1-\left(\frac{2 \pi^{2}}{3^{5 / 4}} \frac{m_{e}}{m_{j}} \frac{Z T_{i}}{T_{e}}\right)^{2 / 3}\right]
$$

\begin{tabular}{|c|c|c|c|c|}
\hline Ros. Corr. & $T_{j}(\mathrm{keV})$ & $\mathrm{T}_{\mathrm{e}}$ (keV) & $\beta(\%)$ & $P_{\text {total }}\left(\mathrm{kW} / \mathrm{m}^{3}\right)$ \\
\hline vio $(1.0)$ & 93.4 & 69.1 & 23.7 & 294 \\
\hline Yes $(0.984)$ & 103.0 & 73.2 & 25.6 & 326 \\
\hline Effect & $+10.3 \%$ & $+5.9 \%$ & $+8.0 \%$ & $+10.9 \%$ \\
\hline
\end{tabular}

Bu11. Am. Phys. Soc. 21, 1114 (1976): 


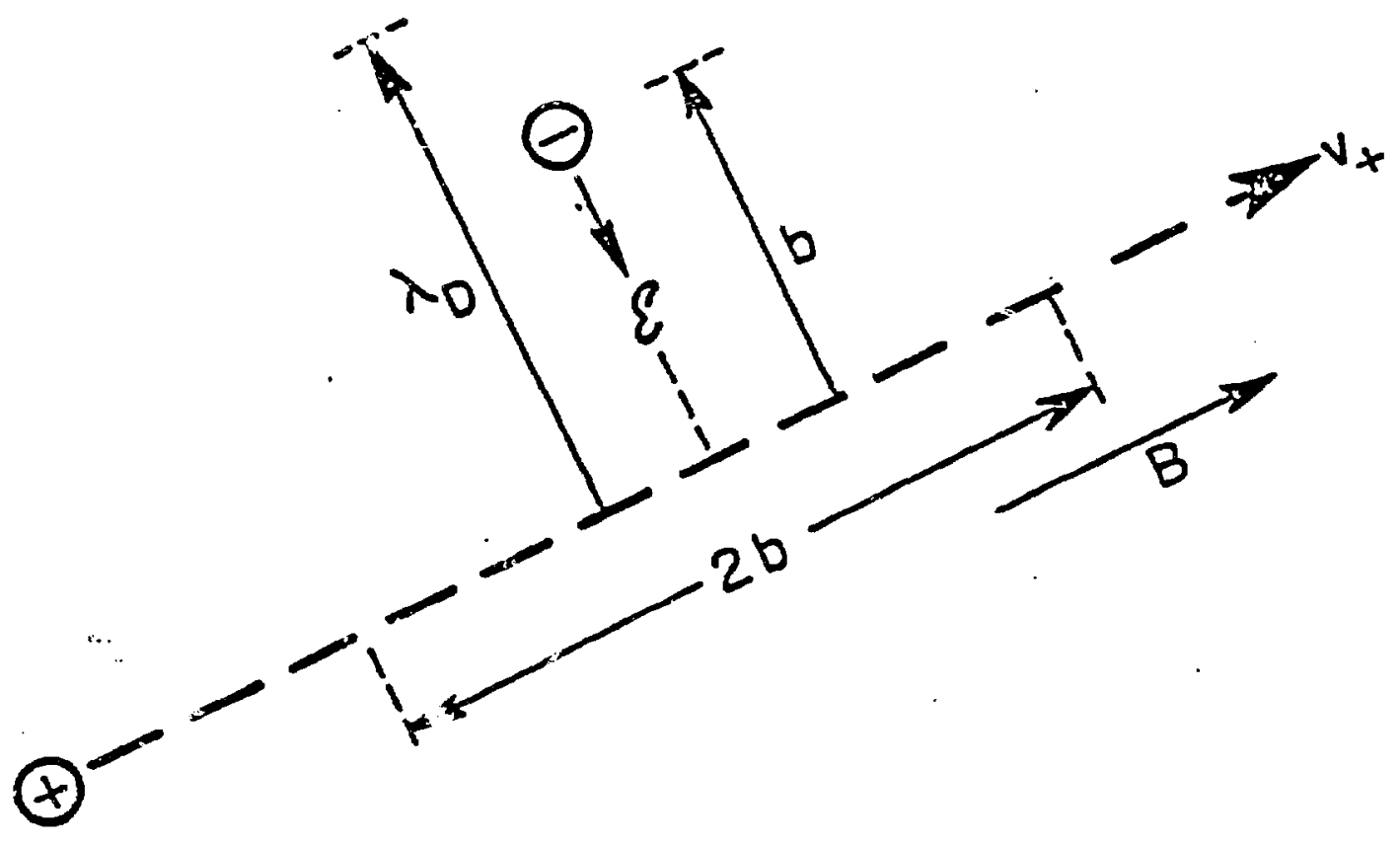

Bohr-Fermi method of analyzing maximum impact parameter for test ion moving at velocity $v_{+}$in an electron sea immersed in a magnetic field, $B$. When the Debye distance, $\lambda_{D}$, is larger than $v_{+} / \omega_{c e} \sqrt{2}$ the latter defines an approximate maximum impact parameter. $\mathcal{E} \sim \mathrm{Ze} / \mathrm{b}^{2}$ and approximate collision time is $2 \mathrm{~b} / \mathrm{v}_{+}$. 


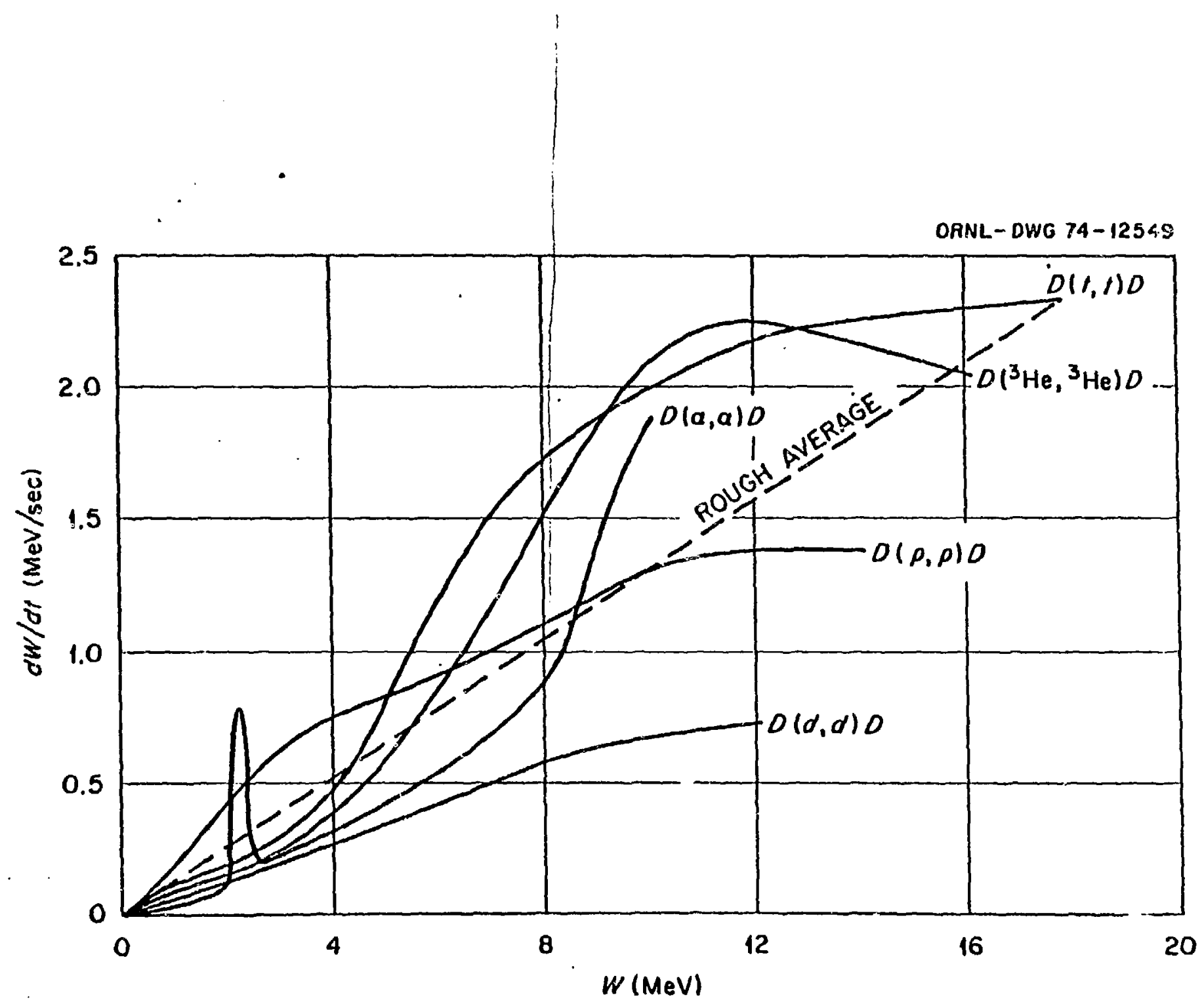

Slowing Down Rates for Nuclear Elastic Collisions with Deulerons. 


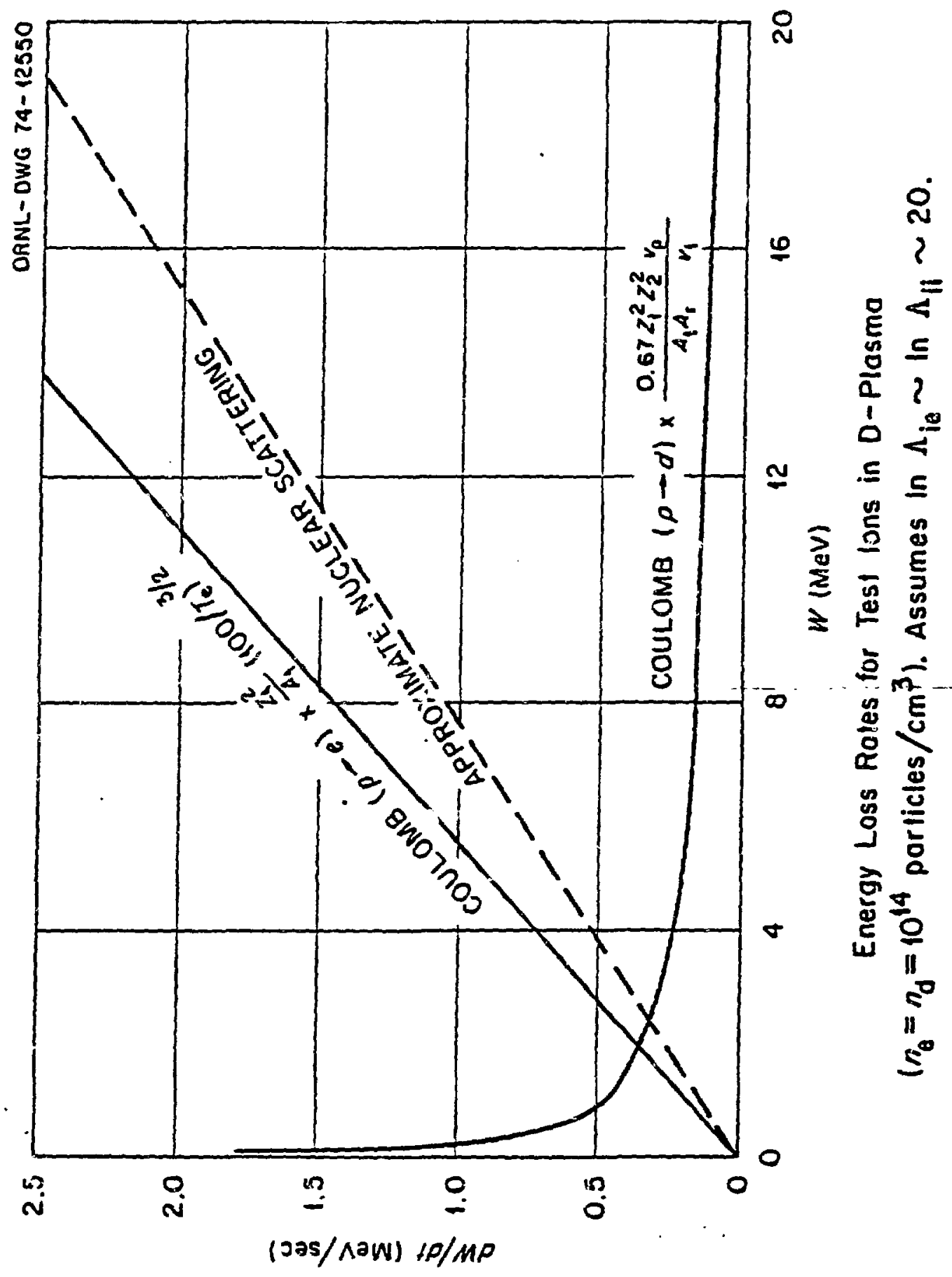

Fig. 14 


\section{NUCLEAR ELASTIC PLUS INTERFERENCE \\ CROSS SECTIONS}

- J. J. Devaney and M. L. Stein, Nuc1. Sclence and Engineering 46, 323 (1971). (5 graphs). $\mathrm{p}, \mathrm{d}, \mathrm{t},{ }^{3} \mathrm{He}, \alpha$ on $\mathrm{d}$.

- S. T. Perkins and D. E. Cullen, Nucl. Sclence and Engineering 77, 20 (1981). (25 graphs). $p, d, t,{ }^{3} \mathrm{He}, \alpha$ with each other.

- S. T. Perkins and D. E. Cullen, UCRL-50400, Vol. 15, Part F (1980). (25 data). $p, d, t,{ }^{3} \mathrm{He}, \alpha$ with each other. 


\section{PROBABILITY OF PROPAGATION CHAIN IN DT}

$$
\begin{aligned}
& \text { Propagation Chain }\left\{\begin{array}{l}
d+{ }^{3} \mathrm{He} \rightarrow \mathrm{p}+\alpha+18.4 \mathrm{MeV} \\
\mathrm{p}+\mathrm{t} \rightarrow{ }^{3} \mathrm{He}+\mathrm{n}-0.8 \mathrm{MeV}
\end{array}\right. \\
& \text { Branching Chain } \quad \mathrm{p}+\mathrm{d} \rightarrow \mathrm{p}^{\prime}+\mathrm{p}+\mathrm{n}-2.2 \mathrm{MeV}
\end{aligned}
$$


CORRECTIONS TO CATALYZED D-D BURNS*

\begin{tabular}{|c|c|c|c|c|c|}
\hline Parametric & $\begin{array}{l}\text { Cat. } \\
\text { D-D }\end{array}$ & $\begin{array}{ll} & \text { Plus } \\
\text { B Effect } & \text { Effer } \\
\end{array}$ & $\begin{array}{c}\text { P1us } \\
\text { Rosen. Effect }\end{array}$ & $\begin{array}{l}\text { Plus } \\
\text { N. S. }\end{array}$ & $\begin{array}{l}\text { Plus } \\
P+T\end{array}$ \\
\hline $\mathrm{T}_{1}(\mathrm{keV})$ & 100 & 143 & 144 & 173 & 175 \\
\hline $\mathrm{T}_{\mathrm{e}}(\mathrm{keV})$ & 82 & 95 & 96 & 101 & 102 \\
\hline$B(\%)$ & 27.3 & 35.3 & 35.5 & 40.3 & 40.7 \\
\hline $\mathrm{P}_{\mathrm{CHP}}\left(\mathrm{kW} / \mathrm{m}^{3}\right)$ & 235 & 334 & 337 & 397 & 407 \\
\hline$P_{\mathrm{NAB}}\left(\mathrm{kW} / \mathrm{m}^{3}\right)$ & 209 & 296 & 299 & 352 & 351 \\
\hline$n_{T} / n_{e}$ & 0.0088 & 0.0140 & 0.0142 & 0.0181 & 0.0178 \\
\hline
\end{tabular}


TRITIUM PRODLCTION TN

D-T RFACTORS WITH DEPLETED TRITIUM

\begin{tabular}{|c|c|c|c|c|}
\hline \multirow{2}{*}{$\begin{array}{c}\text { Operating } \\
\text { Temperature }\end{array}$} & \multicolumn{3}{|c|}{ T Produced / T Consumed $*$} & \multirow{2}{*}{$\begin{array}{c}\text { Required } \\
\mathrm{T} \text { Breeding } \\
\text { Ratios }\end{array}$} \\
\hline & $\left(\mathrm{n}_{\mathrm{T}} / \mathrm{n}_{\mathrm{D}}=1\right)$ & $\left(\mathrm{n}_{\mathrm{T}} / \mathrm{n}_{\mathrm{D}}=0.1\right)$ & $\left.\mathrm{n}_{\mathrm{T}} / \mathrm{n}_{\mathrm{D}}=0.02\right)$ & \\
\hline $30 \mathrm{keV}$ & $0.36 \%$ & $3.6 \%$ & $18 \%$ & $1.00 / 0.96 / 0.82$ \\
\hline 60 & 0.71 & 7.1 & 35.5 & $0.99 / 0.93 / 0.64$ \\
\hline 90 & 1.2 & 12 & i0 & $0.99 / 0.88 / 0.40$ \\
\hline 120 & 1.7 & 17 & 85 & $0.98 / 0.83 / 0.15$ \\
\hline 150 & 2.3 & 23 & 115 & $0.98 / 0.77 / 0.00$ \\
\hline 180 & 3.0 & 30 & 150 & $0.97 / 0.70 / 0.00$ \\
\hline
\end{tabular}

*T Produced $\simeq \frac{1}{2} n_{D}^{2}\langle\sigma v\rangle$ DDt

$\mathrm{T}$ Consumed $\approx \mathrm{n}_{\mathrm{D}} \mathrm{n}_{\mathrm{T}}\langle\sigma \mathrm{v}\rangle \mathrm{DT}$

$\mathrm{T} \operatorname{Prod} / \mathrm{T}$ Cons $\simeq \frac{\mathrm{n}_{\mathrm{D}}\langle\sigma v\rangle \mathrm{DDt}}{2 \mathrm{n}_{\mathrm{T}}\langle\sigma v\rangle \mathrm{DT}}$ 


\section{CAT-DD FUELED REACTORS}

\section{ADVANTAGES:}

1. Lowest fuel cost; gaseous fuel and ashes.

2. Modest total tritium inventory ( $\sim \mathrm{Ig}$ ); no Li blanket.

3. Optimal selection of primary heat exchanger and structures.

4. Fissile and ${ }^{3}$ He tilel breeding.

5. About $45 \% 14 \mathrm{MeV}$ neutrons as DT.

6. Steady-state burn prospect.

\section{DISADVANTAGES:}

1. Rapid isotopic separation and fuel make-up.

2. Total neutron flux comparable to DT.

3. Requires higher temperatures and $n \tau$ 's than DT.

4. Requires $\beta \gtrsim 20 \%$ for economic burn.

5. Major safeguards problem (neutrons are "free"). 
NUCLEAR EFFECTS

1. Nuclear elastic scattering of fuel ions to suprathermal energies

$$
\left(x_{\text {fast }}+d+X^{+}+d_{\text {fast }}\right)
$$

2. Nuclear dissociation events

$$
\left(\mathrm{X}_{\text {f ast }}+{ }^{6} \mathrm{LI}+\mathrm{X}^{\prime}+{ }^{6} \mathrm{LI}^{*}(2.2 \mathrm{MeV})+\mathrm{X}^{\prime}+\alpha+\alpha-1.5 \mathrm{MeV}\right)
$$

3. Partition of nuclei among excited nucleav states? ${ }^{1}$

4. Gamma ray production

$$
\left(D+T+{ }^{5} \mathrm{He}^{*}(16.7 \mathrm{MeV}) \stackrel{0.0002}{\rightarrow}{ }^{\mathrm{He}}+\gamma+17 \mathrm{MeV}\right)
$$

5. Nuclear "spin" conservation?

$$
\left[\mathrm{D}(1)+\mathrm{T}\left(\frac{1}{2}\right)+{ }^{5} \mathrm{He}^{*}(3 / 2)+\mathrm{n}\left(\frac{1}{2}\right)+\alpha(0)+17.6 \mathrm{MeV}\right]
$$

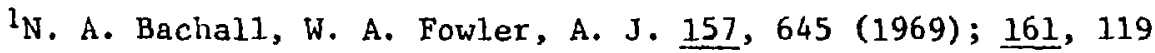
(1970). 


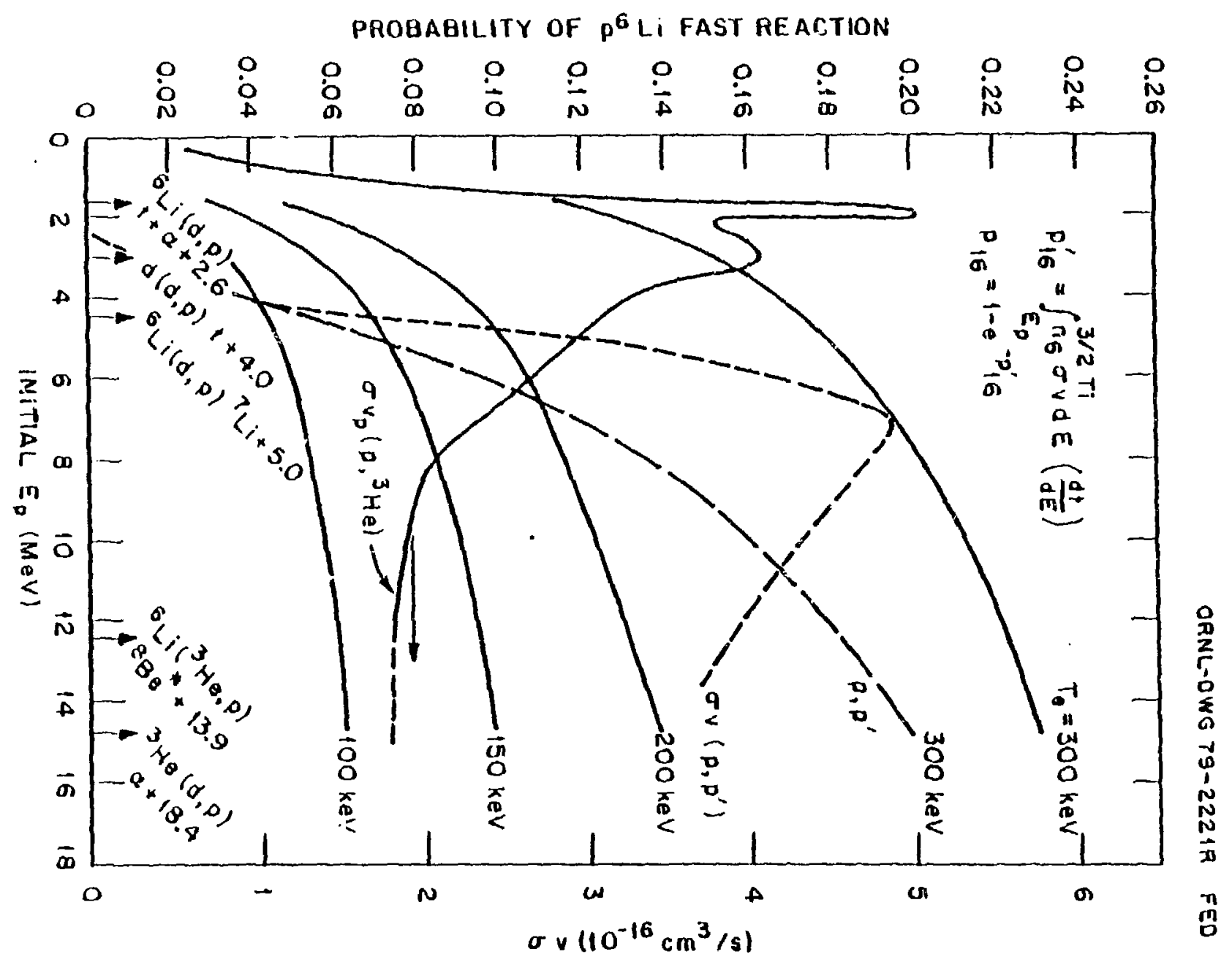




\section{ICRH COUPLING BETWEEN FUSION PRODUCT CHARGED PARTICLES AND FUEL IONS}

- ICRH Heating of plasmas has been demonstrated (primarily by minority species coupling in dense plasmas).

- Selective heating of fuel ions will drive $T_{\mathfrak{i}}>T_{e}$ and enhance reactivity.

- Alphas $(Z / A=1 / 2)$, tritons $(Z / A=1 / 3),{ }^{3} \mathrm{He}$ $(Z / A=2 / 3)$, deuterons $(Z / A=1 / 2)$, protons $(Z / A=1)$.

- What is role of coherent bunching of fusion product ions in ion cyclotron motion?

- Conclusion: Need for in depth study of in situ ICRH coupling in burning plasmas. 


$$
P-1 I_{B} \text { FUEL }
$$

1. Ignition prospects but no steady-state burns yet.

2. Problems of $10_{\mathrm{B}}$ content $\left({ }^{10} \mathrm{~B} /{ }^{1} \mathrm{~B}: 18.7 / 81.3\right)$ and ${ }^{1 l_{B}}$ cost.

$$
\left(p+{ }^{10} \mathrm{~B}+\alpha+{ }^{7} \mathrm{Be}^{*}+1.147 \mathrm{MeV}\right)
$$

3. Problems of condensable ashes (debris)

$$
\text { ( } 2 \text { tons/GW th } y \text { ) }
$$

4. Synchrotron radiation problem

(FRM, Ion Layer, SURMAC) 
RADIOACTIVE ${ }^{7} \mathrm{Be}$ PRODUCTION IN $\mathrm{P}-10,11_{\mathrm{B}}$ FUEL CYCLE*

$$
\begin{aligned}
& \mathrm{P}+{ }^{11} \mathrm{~B} \rightarrow 3^{4} \mathrm{He}+8.664 \mathrm{MeV} \\
& \mathrm{P}+{ }^{10} \mathrm{~B} \rightarrow{ }^{7} \mathrm{Be}+{ }^{4} \mathrm{He}+1.150 \mathrm{MeV} \quad(\varepsilon, \gamma: 0.5 \mathrm{MeV}, 12 \%)
\end{aligned}
$$

\begin{tabular}{|c|c|c|c|}
\hline \multirow[b]{2}{*}{$\mathrm{T}$} & \multicolumn{2}{|c|}{$<\sigma v>\left(\mathrm{m}^{3} / \mathrm{s}\right)$} & \multirow{2}{*}{$\begin{array}{c}{ }^{7} \mathrm{Be} \\
\text { Production }^{\dagger}\end{array}$} \\
\hline & $\mathrm{P}+{ }^{11} \mathrm{~B}$ & $\mathrm{P}+{ }^{10} \mathrm{~B}$ & \\
\hline $200 \mathrm{keV}$ & $1.67 \times 10^{-22}$ & $2.45 \times 10^{-23}$ & 0.0045 \\
\hline 300 & $2.43 \times 10^{-22}$ & $5.96 \times 10^{-23}$ & 0.0076 \\
\hline 400 & $2.76 \times 10^{-22}$ & $9.46 \times 10^{-23}$ & 0.0106 \\
\hline
\end{tabular}

$t_{10_{B} /{ }^{1} I_{B}}=3 / 97$

*other side reactions needing evaluation:

${ }^{4} \mathrm{He}+10_{\mathrm{B}}+13 \mathrm{~N}+\underline{\mathrm{n}}+1.073 \mathrm{MeV}$

${ }^{4} \mathrm{He}+10_{\mathrm{B}}+{ }^{12} \mathrm{C}+\underline{D}+1.355 \mathrm{MeV}$

$\mathrm{X}+11_{\mathrm{B}}+\mathrm{X}^{+}+11_{\mathrm{B}}{ }^{*}-2.125 \mathrm{MeV}(\gamma$ emission $)$

$\mathrm{D}+{ }^{11_{\mathrm{B}}}+{ }^{12} \mathrm{C}+\underline{\mathrm{n}}+13.731 \mathrm{MeV}$

$\mathrm{X}+{ }^{7} \mathrm{Be}+\mathrm{X}^{-}+{ }^{3} \mathrm{He}+{ }^{4} \mathrm{He}-1.586 \mathrm{MeV}$ 
THERMAL AND DENSITY EXCURSIONS IN IGNITED PLASMAS

- Fusion plasmas have positive temperature coefficients (unstabie) at ignition point.

- Fusion plasmas have negative temperature coefficient (stabie) at burning temperature.

- Fusion plasmas have positive density coefficient at burning temperature.

- Power excursion following ignition may unload fuel absorbed on and adsorbed in first wall leading to further power excursion.

- Conclusion: Need for in depth study of transients in ignited plasmas. 
SUMMARY

1. Our understanding of reacting fusion fuels shows remarkable progress.

2. There is a need for more plasma and nuclear physics input to improve our understanding of reacting fusion fuels. 